\title{
FEMTOSECOND SPECTROSCOPY OF THE FIRST EVENTS OF THE PHOTOCHEMICAL CYCLE IN BACTERIORHODOPSIN
}

\author{
M C. NUSS, W ZINTH, W. KAISER \\ Physik-Department der Technischen Universutōt München, Munch, West Germany \\ E. KÖLLING and D. OESTERHELT \\ Max-Planck-Itstitut für Bıochemie, Mariursried near Munich, West Germany
}

Received 21 March 1985

\begin{abstract}
The first steps in the photochemistry of bacteriorhodopsin (BR) are investigated with light pulses of 160 fs duration. Four samples are studied: (i) the purple membrane, (ii) deuterated puple membrane, (iii) BR trimers and (iv) BR monomers In all saroples the first intermediate $J$ is formed within $430 \pm 50$ fs. No isotope effect is observed in the formation of $J$ upon deutera tigil, in contrast to previous reports with much higher excilation energies. Thus proton movemeni to or from the retinal Schiffs base is not relevant during the first step. Comparing the data for trimeric and monomsirs Bp. suggests an upper limit of 50 fs for the transfer of excilation energy from the excitoncally coupled trimer to a single retingl chromophore.
\end{abstract}

\section{Introduction}

Bacterio rhodopsin (BR) acts as a light-driven proton pump in Halobacterium halobium, converting light energy into an electrochemical gradient across the cell membrane [1]. Thes gradient is subsequently used to synthesize adenosine triphosphate (ATP) and to drive transport processes. In this way bacteriorhodopsin mediates photosynthesis in Halobacteria. Bacteriorhodopsin's primary structure is known. The polypeptide chain of 248 aminoacids folds into seven $\alpha$-helical segments each spanning the entire cross section of the membrane. Retinal forms a protonated Schiff's base with one lysine 216 of the polypeptide chain. In the light-adapted state, all retinal molecules have the alltrans configuration, while the dark-adapted pigment is a $50 \%$ mieture of all-trans and 13-cis, 15-cis chromophores [2].

In most halobacterial strains BR forms trimers which are arranged in a two-dimensional hexagonal lattice within the cell membrane. The patches formed can be isolated by centrifugation techniques upon lysis of the halobacterial cells and fragmentation of the cell membrane by water. The isolated patches are called purple membranes. Within the trimers the BR molecules are excitonically coupled, as seen by the b1lobe type of the CD spectrum. Specific treatments of purple membranes with detergents such as octylglucoside in high salt destroy the crystalline lattice but preserve an oligomeric, presumably the trimeric, state. Other detergents such as Triton $X 100$ monomerize BR completely [3].

After excitation of the light-adapted pigment, bacteriorhodopsin undergoes a photochemical cycle of approximately $10 \mathrm{~ms}$ duration. During the cycle the intermediates $J, K, L, M$ and $O$ are formed, which can be distinguished by their absorption bands $[4,5]$. The changes of the atsorption properties are accompanied by proton movements (deprotonation and protonation of the Schiff's base), by configurational changes of the retinal molecule and by conformational changes of the protein $[6,7]$. Experimental evidence exists that isomerisation of the all-trans retinal to 13-cis retinal occurs in the first step, i.e. the formation of $J$ [8-10]. There are also strong indications that isomerisation is essential for the formation of the first groundstate intermediates in BR $[10,11]$.

The first events of the photochemical cycle of bac- 
teriorhodopsin have been observed in a number of publications [12-17]. Unfortunately, contradictory results were found for the picosecond events of $B R$, e.g., a deuterium effect, reported by some authors, could not be confirmed by others $[13,16,17]$. Most of the earlier experiments used very high excitation energies, where more than one photon per BR molecule was absorbed during the time of the excitation pulse. Under these conditions non-physiological reaction channels may occur Recent experiments with low excitation energies gave no indication of differences in the kinetics between deuterated and undeuterated samples on the picosecond time scale (i.e. the formation of the K state) [16].

In this Letter we report on the very first steps of the photochemical cycle of bacteriorhodopsin with a time resolution of better than $50 \mathrm{fs}\left(5 \times 10^{-14} \mathrm{~s}\right)$ and low excitation energies. Emphasis is put on the formation of the intermedrate $J$ under conditions of protonation and cleuteration. Furthermore, BR in the crystalline trimeric and monomeric states are compared to analyze the energy transfer from the excitonically coupled BR trimer to a single retinal chromophore.

\section{Sample preparation}

Purple membranes (PM) were prepared from Halobacterium halobium according to the procedure given in ref [18] and the membranes suspended in $0.01 \mathrm{M}$ potassium phosphate buffer, $p H$ 7. Deuterated samples were prepared by suspending the membranes in $D_{2} O$ and lluminating the suspension with light from a 900 $W$ Xe lamp $(\lambda>520 \mathrm{~nm})$. After 30 min the membranes were sedimented by centrifugation $(30 \mathrm{~mm}$, $50000 \mathrm{~g}$ ). This procedure was repeated twice The deuterated samples were kept in $0.01 \mathrm{M}$ deuterated potassium phosphate buffer. The exchange of the hydrogen at the Schiff's base nitrogen by a deuterium was confirmed with resonance Raman spectroscopy.

BR trimers were prepared by stirring PM (32 $\mu \mathrm{M}$ ) in $1 \mathrm{M} \mathrm{NaCl}$ containing $10 \mathrm{mM}$ morpholinopropanesulfonic acid (MOPS), $p H 7$, and $3 \%$ octyl- $\beta$-D-glucopyranoside (OG) for three days at room temperature. The sample was then diluted to $1 \%$ OG with $10 \mathrm{mM}$ MOPS $p H 7$ in $1 \mathrm{M} \mathrm{NaCl}$ and concentrated in an Amicon cell to a final concentration of $480 \mu \mathrm{M}(1 \mathrm{M}$ $\mathrm{NaCl}, 10 \mathrm{mM}$ MOPS, 1\% OG) and centrifuged for $1 \mathrm{~h}$ at $200000 \mathrm{~g}$ and $10^{\circ} \mathrm{C}$. BR trimers at a concentration of $320 \mu \mathrm{M}$ (20 OD) were thus obtained.

Monomers were prepared by stirring PM (400 $\mu \mathrm{M})$ in $10 \mathrm{mM}$ N-2-hydroxyethylpiperazine- $\mathbf{N}^{\prime}$-ethanesulfonic acid (HEPES), $p H 6$ or $p H 7$ containing $30 \mathrm{mg} / \mathrm{ml}$ Triton $X 100$ and $001 \%$ sodium azide for two days at $34^{\circ} \mathrm{C}$. After centrifugation (see above) a BR monomer solution of about $320 \mu \mathrm{M}$ BR was obtained [3]. Samples at $p H 6$ and $p H 7$ showed identical behaviour in $C D$ spectroscopy and in the femtosecond experuments.

The exact state of aggregation of BR in the purple membrane (PM), the BR trimers and the BR monomers in suspension or solution is not precisely known. Therefore characteristic features are used for definition of these states:

Purple membrances (PM) are membrane patches as isolated in the procedure described above. They show characteristic diffraction patterns in $\mathrm{X}$-ray deffraction experiments

Purple membranes in $\mathrm{D}_{2} \mathrm{O}\left(\mathrm{PM}_{\mathrm{D}_{2} \mathrm{O}}\right)$ contain membrane patches as $\mathrm{PM}$, but the hydrogen at the protonated Schiff's base is exchanged by deuterium This is verified by resonance Raman spectroscopy.

BR trimers contain trimers or trimer aggregates of BR which cannot be pelleted under the centrifugation conditions described above. They should not show the diffraction patterns found for PM but the same excitonic coupling is found for the trimenc BR and for the purple membrane PM, as analyzed by $C D$ measurements (bi-lobe spectra with a maximum at $545 \mathrm{~nm}$ and $a$ minimum at $596 \mathrm{~nm}$ ). Furthermore these probes show no non-linear absorption as described below in section 4.

BR momoners contain monomers or monomer aggregates of BR and are simular to BR trimers, but excitonic coupling is lacking (no bi-lobe in CD spectra, $\lambda_{\text {max }}$ at $564 \mathrm{~nm}$ ).

All samples had an optical density of about 20 at $580 \mathrm{~nm}$ and were placed in a cell of $300 \mathrm{~mm}$ thickness. Prior to each experimental scan the sample was illumlnated with actinic light $(\lambda>500 \mathrm{~nm})$ for $30 \mathrm{~min}$ to ensure light adaption. Additional illumination for 5 min was given between the individual scans (duration 3 min). 


\section{Experimental}

A schematic representation of the apparatus is shown in fig. 1. A colliding pulse mode-locked (CPM) dye laser [19] yielded pulses of $110 \mathrm{fs}$ duration at $\lambda$ $=620 \mathrm{~nm}$ at a repetition rate of approximately 100 MHz. Transient absorbance changes were studied by the excite-and-probe technique. Since the cycle time to the bacteriorhodospin photocycle is about $10 \mathrm{~ms}$, the repetition rate of the CPM laser had to be reduced ual pulses with a Pockels cell (tnggered with a repenot intermediate states of the photocyrcle. This was accomplished (i) by selecting pulse trains of 5 individual pulses with a Pockels cell (triggered with a repetition rate of $100 \mathrm{kHz}$, corresponding to one pulse train per $10 \mu \mathrm{s}$ ), (ii) by using a rotating cuvette ( 25 $\mathrm{Hz}$ ) with a distance of $50 \mathrm{~mm}$ between the sample and the center of rotation, and (iii) ty focusing pump and probe beams to a spot $<20 \mu \mathrm{m}$ diameter in the sample. This guaranteed that each pulse train illumi-

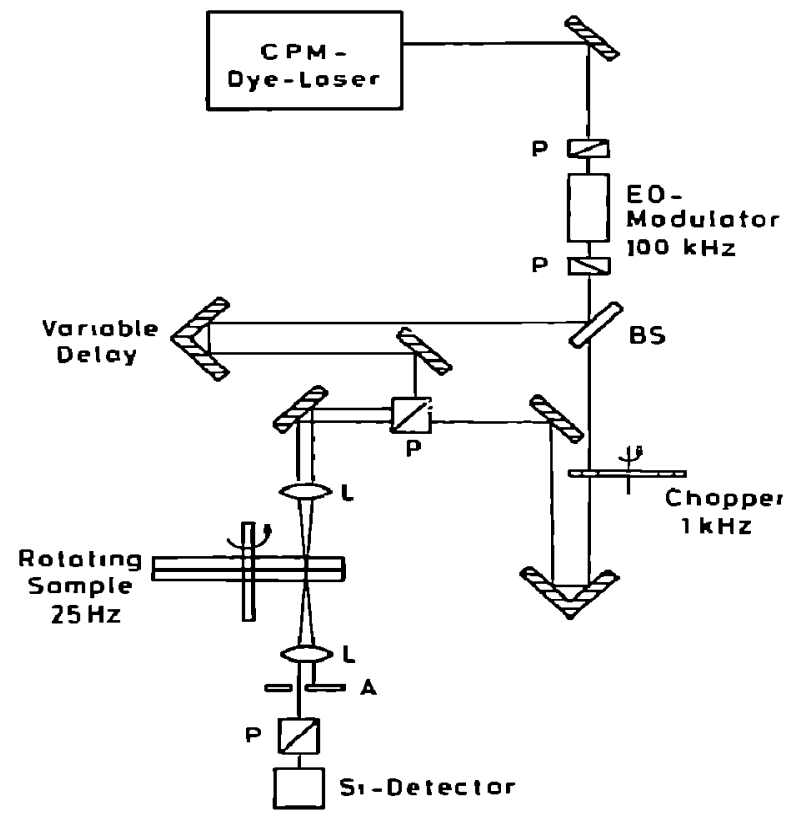

Fig. 1. Schematic representation of the apparatus for femtosecond pulse spectroscopy. Pulse trains containing 5 pulses each were selected by an electro-optical (EO) modulator at a rate of $100 \mathrm{kHz}$. The sample rotated in a cuvette at $25 \mathrm{~Hz}$ The exciting and probing pulse trains were selected and projected onto the sample with polarzers $P$, beam splitters BS and lenses $L$ The exciting beam was stopped at the aperture $A$. nated a new portion of the sample (angular velocity: $75 \mu \mathrm{m} / 10 \mu \mathrm{s})$ and that, after one revolution of the rotating cuvette ( $40 \mathrm{~ms})$, all intermediate photoproducts har been reconverted to the starting species, ie. the light-adapted BR.

The pulse trains from the Pockels cell were split by a beam splitter into a strong exciting and a much weaker probe beam. The interrogating probe pulse was delayed by a delay line which consisted of a corner cube reflector mounted on a translator, driven by a stepping motor (resolution of the stepping motor is $6.7 \mathrm{fs}$ ). The probe beam passed a half-wave plate polarizing the probe beam perpendicularly to the exciting beam. Both beams were recombined at a polarizing beam splitter and were focused non-collinearly onto the rotating sample cuvette. The absorbance changes unduced by the exciting pulse were then monitored by the probe beam. The transmission change was directly measured with a silicon detector and a phase-sensitive amplifier locked to the frequency of a light chopper $(1 \mathrm{kHz})$ placed in the exciting beam. Stray light from the exciting beam was strongly reduced by a second polarizer in front of the detector cell (fig. 1).

The induced absorbance changes as a function of the delay time $t_{D}$ between exciting and probing pulses were averaged over several scans. The overall time resolution was deduced from the measurement of the cross correlation trace between exciting and probing pulses. These data were obtained by replacing the sample by a thin $(0.1 \mathrm{~mm})$ non-linear crystal. The pulse duration was found to be increased from 110 to $160 \mathrm{fs}$ by the dispersion in the Pockels cell. Time zero was determined by the peak of the cross-correlation trace.

A constant background had to be subtracted from the measured absorbence change. This background resulted from the fact that not single pulses but pulse trains containing 5 pulses were used. Each pulse promotes a very small fraction of the BR molecules into the $K$ intermediate causing a constant background when the next pulse of the same train arrives.

The intensity of the exciting pulses at the sample was kept very low. Within one pulse train only, less than $0.2 \%$ of the BR molecules absorbed a photon and entered the photochemical cycle. The maxumal absorbance changes were only $\Delta A=10^{-4}$ in all samples. 


\section{Results}

The experiments were carried out with pulses of $\lambda$ $=620 \mathrm{~nm}$. At this wavelength bacteriorhodopsin as well as the first photoproducts $J$ and $K$ absorb [16]. As a consequence a complex time dependence of the absorbance change is expected. Indeed we find four different phenomena occurring within the first five ps $\left(5 \times 10^{-12} \mathrm{~s}\right)$. Fig. 2 a shows the data from purple membranes suspended in $\mathrm{H}_{2} \mathrm{O}$ : (i) A strong peak of induced absorption occurs at time zero with a width of 100 fs corresponding to the coherence time of the

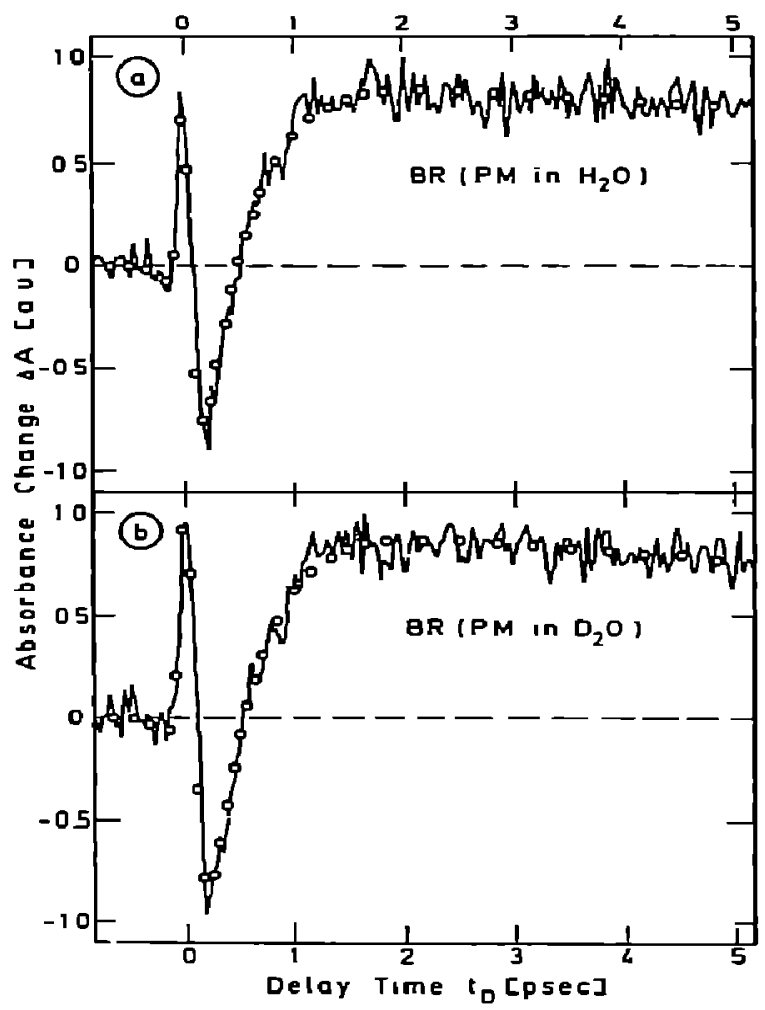

Fig 2 Transient absorbance changes of bacteriorhodopsin in purple membranes suspended in (a) $\mathrm{H}_{2} \mathrm{O}$ and (b) $\mathrm{D}_{2} \mathrm{O}$. Exciting and probing pulses of $160 \mathrm{fs}$ duration and at $\lambda=620 \mathrm{~nm}$ were used. Open circles indicate least-squares fits of a rateequation system to the data with time constants of $430 \mathrm{fs}$ and 5 ps. The same tume constants are obtained for the deuterated and undeuterated samples. The maximal absorbance changes correspond to $\triangle A=10^{-4}$. The 5 ps kinetres is not seen in the monomer sample because it is covered by the 1 ps time constant light pulses. (ii) Subsequently, rapid bleaching (ie. reduced absorption) is observed. (iii) At times $t_{D}>$ 03 ps the absorption increases with a time constant of $430 \pm 50 \mathrm{fs}$ (iv) A slow ( $\approx 5 \mathrm{ps}$ ) and small relative absorbance decrease leads to an absorbance level which remains constant for at least 30 ps (not shown in the figure).

Fig. $2 b$ shows - on the same time scale - the timedependent absorbance changes of purple membranes suspended in $D_{2} O$, where the Schiff's base at the retinal-lysine linkage has been deuterated. No differences of time constants and amplitudes can be seen with respect to the undeuterated sample.

In addition to $B R$ in the purple membrane state, we investigated solubilized $B R$ in the trimeric and monomeric state (see definition in section 2) $\mathrm{BR}$

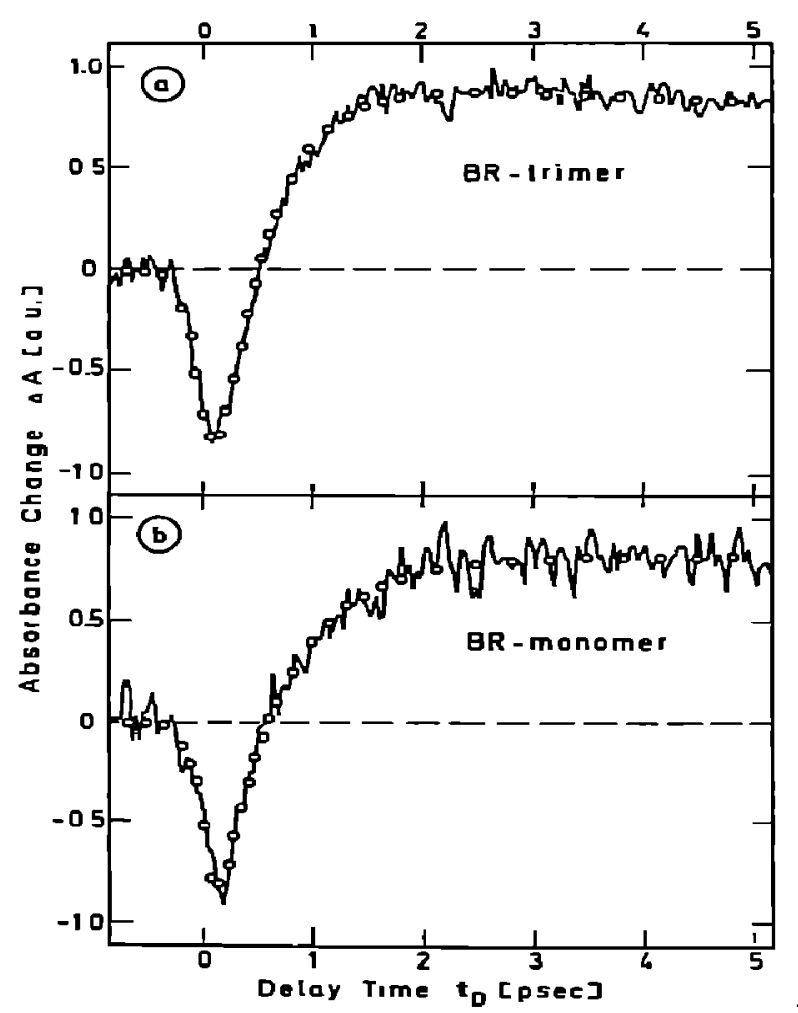

Fig 3. Comparison of transient absorbance changes in: (a) BR trmers and (b) BR monomers. The numerical fits (open circles) yield time constants of $430 \pm 50 \mathrm{fs}, 5 \pm 1$ ps for the trimer and $0.4 \pm 0.1 \mathrm{ps}, 1.0 \pm 0.3 \mathrm{ps}$ for the monomer samples. $\Delta A_{\max }=10^{-4}$. 
trimers do not form a two-dimensional hexagonal lattice but excitonically couple the threc retinal chromophores. In monomeric BR this interaction between the 3 BR proteins is lost. Fig. 3 shows the absorbance kinetics of trimeric (fig. 3a) and monomeric (fig. 3b) BR. The most striking difference to the data from BR in the purple membrane state (fig. 2) is the disappearance of the peak at time zero for both trimeric and monomeric BR. At delay times $t_{\mathrm{D}}>0.3 \mathrm{ps}$ the same kinetics (time constant $430 \pm 50 \mathrm{fs}$ ) are found for the BR trimers (sec fig. 3a) as for the purple membrane (see fig. 2). In the case of the BR monomers the situation is somewhat different two processes with time constants of $0.4 \pm 0.1$ ps and $1.0 \pm 0.3 \mathrm{ps}$ occur.

\section{Discussion}

The slowest kinetics we observed in all samples has a time constant of 5 ps and leads from a state of strong absorption to one of weaker absorption Since the $K$ state was reported to be formed within $5 \pm 1$ ps [16], the "final" state on our time scale is certainly the $K$ state The preceding absorption is attributed to the first intermediate, the $J$ state.

The photoproduct $J$ is formed as a result of the decay of the preceding excited singlet state $S_{1}$ of $B R$ This transition was not resolved in previous experiments. From a fit of picosecond experiments a time constant of $0.7 \pm 0.3$ ps was estimated [16]. The absorbance curves of fig. 2 show, clearly resolved, the electronically excited state $S_{1}$, with reduced absorption compared to the BR ground state, and subsequently the reappearance of the ground-state intermediate $J$ with a time constant of $430 \pm 50$ fs.

The measurements of $B R$ in the purple membrane (fig. 2) display at time zero a strong peak of increased absorption There at several indications that this peak is not due to a real molecular transition but is caused by a non-linear process. We recall that the cross correlation of the femtosecond pulses is measured in a nonlinear crystal. Two equal pulses interact in the crystal and the generation of the second harmonic at $310 \mathrm{~nm}$ is observed as a function of the delay between the two pulses. It is important that second-harmonic generation is only possible in media which do not have inversion symmetry on the length scale of one wavelength. Similarly, we observed radiation of the second hamonic at $\lambda=310 \mathrm{~nm}$ in the purple membrane state which has a hexagonal lattice structure without an inversion symmetry and a diameter of about 0.5 $\mu \mathrm{m}$. On the other hand, we could not observe secondharmonic radiation in the isolated trimers and monomers, which are much smaller. This second-hamonic generation pretends an induced absorbance in the excite-and-probe experiment of the purple membrane at time zero and is a result of the crystallinic array of the chromophores in the purple membrane.

In the purple membrane the three symmetrically arranged BR molecules are excitonically coupled [20]. After light excitation the absorbed energy is first delocalized over the trimeric BR unit This energy will be trapped subsequently by one of the three retinal chromophores in the trimer which may cause absorption changes and may delay the next steps of the photochemistry. Therefore, one has to consider an additional fast process. With the exception of the spike at time zero the femtosecond kinetics demonstrate the same properties of trimeric BR (fig 3a) as $B R$ in the purple membrane (fig. 2a): the same time constant of $430 \pm 50$ fs for the formation of the $J$ intermediate is found The fit of the data for monomeric BR (fig. 3b) shows two tume constants of $0.4 \pm 0.1$ ps and $1.0 \pm 0.3 \mathrm{ps}$ in the formation of a state with increased absorption We attribute the longei time constant to a considerable anount (38\%) of 13-cis retinal molecules which are present even in the light-adapted specimen [21]. Thus we assign the 1 ps kunetics to the 13-cis retinal, ie. to the BR dark cycle. Disregarding this process, we found within the experimental accuracy no retardation of $\mathbf{J}$ formation for the trimer samples with respect to monomers. Thus we have a strong indication that the energy irapping process from excitonically coupled trimer excited states to single retinal chromophores occurs within $50 \mathrm{fs}$

The subpicosecond experiments presented here give new information on the molecular processes occurring in BR after optical excitation: The absence of an isotope effect in the steps $S_{1} \rightarrow J$ and $J \rightarrow K$ is a strong indication that proton transfer is not the primary process of the photochemical cycle of BR $\neq$.

* A recent paper showed an indication for a deuterium effect even in the very early steps [17]. This seems to be due to a fit to the experimental data neglecting absorbance differences between the intermediates $J$ and $K$. 


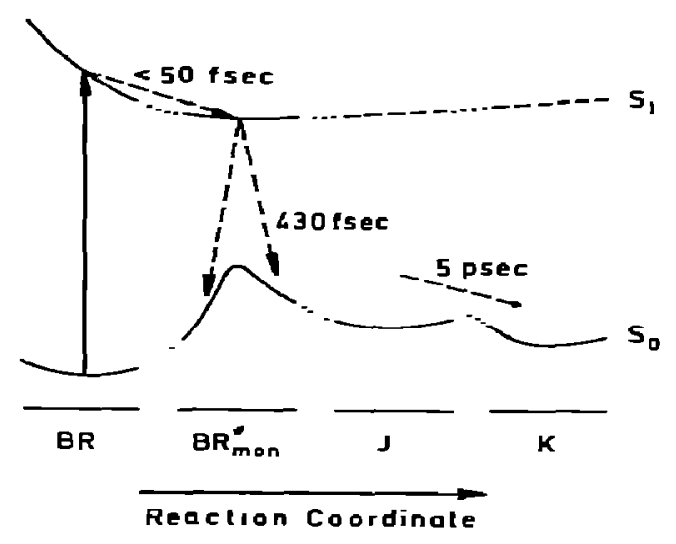

Fig 4. Schematic model of the ground-state $\left(S_{0}\right)$ and excitedstate $\left(S_{1}\right)$ encrgy surfaces of bacteriorhodopsm as a function of the reaction coordinate. Photochemistry is unitiated by absorption of a photon by the BR molecule. The excitation energy is trapped in the excited state of a single BR chromophore BR mon. Within 430 fs the molecule decays back into either the BR ground state or the intermediate J. The subsequent $J \rightarrow K$ transition is a ground-state process with a time constant of $5 \mathrm{ps}$

On the other hand, the strong similanity of the absorption spectra of $K$ and $J$ and the experimental findung that $\mathrm{K}$ contains a 13-cis retinal $[8-10,16]$ support the interpretation that $J$ already contains 13 cis retinal. As a consequence, isomerisation must proceed very rapidly withun $430 \pm 50$ fs. This short time constant is not surprising in the light of other experiments showing similar rapid isomerisation processes [22].

The molecular events are summanzed in a schematic energy surface diagram (fig. 4). The absorbed photon promotes BR in an excited delocalized state $\mathrm{BR}^{*}$ of the trimer unit. The energy is subsequently trapped in the excited state of a single monomer BR $_{\text {mon- }}^{*}$ From the bottom of the $S_{1}$ potential surface, partition into the original ground state and into the isomerized 13-cis, 14-s-cis ground state (the $J$ intermediate) takes place within $\mathbf{4 3 0}$ fs. Apparently there is no energy barrier against isomerisation in the cxcited state. The $J \rightarrow K$ transition is a ground-state process, where probably the rearrangement of the protein (relaxation of the oligomethylene chain of lysine 216 leads to the absorption changes with a time constant of 5 ps.

\section{Conclusions}

In this Letter we were able to describe the formation of the first intermediate $J$ in the photocycle of bacteriorhodopsin under low excitation conditions and with 50 fs time resolution. The product is formed with a time constant of $430 \pm 50$ fs. The kinetics is not sensitive to deuteration of the Schiff's base. Thus proton transfer cannot be involved in this transition Our experiments strongly support isomerisation taking place in this first step of the photochemical cycle of bacteriorhodopsin. Isomerisation starts on the excitedstate energy surface of BR and is completed after $\mathbf{4 3 0}$ fs when the ground-state intermediate $J$ is formed.

Differences in the absorbance kinetics of BR monomers and BR trimers exist (assigned to the dark cycle of BR). Energy transfer from the delocalized excited state of the trimer to a single retunal chromophore apparently takes place within less than 50 fs.

\section{References}

[1] D. Oesterhelt and W. Stoeckenius, Proc Natl Acad. Sci US 70 (1973) 2853.

[2] G.S. Harbison, S.O. Smith, J.A Pardoen, C. Winkel, J. Lugtenburg, J. Herzfeld, R. Mathies and R.G. Grifrin, Proc. Natl ACad Sci US 81 (1984) 1706.

[3] N.A. Dencher and M.P. Heyn, Meth Enzymol 88 (1982) 5 .

[4] R.H. Lozier, R.A. Bogomolni and W. Stoeckenius, Biophys. J. 15 (1975) 955.

[5] M.C. Kung, D. Devault, B. Hess and D. Ocstexhelt, Biophys J. 15 (1975) 907.

[6] M. Pettei, A.P. Yudd, K. Nakanishi, R. Henselman and W. Stoeckenius, Biochemistry 16 (1977) 1955.

[7] B. Aton, A.G. Doukas, R.H. Callender, B. Becher and T.G. Ebrey, Biochemistry 16 (1977) 29.

[8] M. Braiman and R. Mathies, Proc. Natl Acad. Sci US 79 (1982) 403.

[9] C.L. Hsieh, M.A. ElSayed, M. Nicol, M Nagumo and J.H. Lee, Photochem. Photobiol 38 (1983) 83.

[10] H.-J. Polland, M.A. Franz, W. Zinth, W. Kaiser, E. Kolling and D. Oesterhelt, Biochim. Biophys Acta 767 (1984) 635.

[11] J.M. Fang, J.D. Carriker, V. BalogF-Nair and $K$ Nakanishi, J. Am Chem Soc 105 (1983) 5162.

[12] K.J. Kaufmann, P.M. Rentzepis, W. Stoeckenius and A. Lewis, Biochen. Biophys. Res Commun 68 (1976) 1109.

[13] M.L. Applebury, K.S. Peters and P.M. Rentzepis, Biophys J. 23 (1978) 375. 
[14] E.P. IPpen, C.V. Shank, A. Lewis and M.A. Marcus, Science 200 (1978) 1279.

[15] Y. Shishida, S. Matuoke, Y. Hidaka and T. Yoshizawa, Biochim. Biophys. Acta 723 (1983) 240.

[16] H.-J. Polland, W. Zinth and W. Kaiser, in: UItrafast phenomena, Vol 4, eds. D.H Auston and K.B. EisenthaI (Springer, Berlin, 1984) p. 456;

H.-J. Polland, A. Franz, W. Zinth, W. Kaiser, E. Kolling and D. Oesterhelt, Biophys. J., to be published.

[17] M.C. Downer, M. Islam, C.V. Shank, A. Harootunian and $A$ Lewis, in- Ultrafast phenomena, Vol 4, eds D.H. Auston and K B. Eisenthal (Springer, Berlin, 1984) p. 500 .
[18] D. Oesterhelt and W. Stoeckenius, Meth. Enzymol 31A (1974) 667

[19] R.L. Fork, B.L Greene and C.V. Shank, Appl Phys Letters 38 (1981) 671.

[20] B. Becher and T.G. Ebrcy, Biochem. Biophys. Res Commun 69 (1976) 1.

[21] R. Casadio, H. Gutowitz, P. Mowery, M. Taylor and W. Stoeckenius, Biochim Biophys Acta 590 (1980) 13.

[22] B.L Greene and R.C. Farrow, J. Chem. Phys 78 (1983) 3336. 